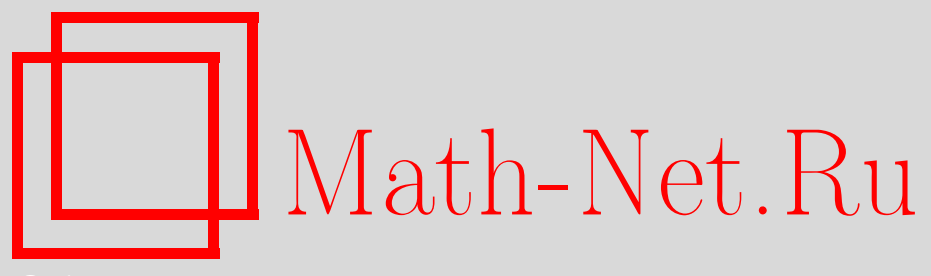

Е. А. Мазепа, О существовании целых решений одного полулинейного эллиптического уравнения на некомпактных римановых многообразиях, Матем. заметки, 2007, том 81, выпуск 1, 153-156

DOI: https://doi.org/10.4213/mzm3529

Использование Общероссийского математического портала Math-Net.Ru подразумевает, что вы прочитали и согласны с пользовательским соглашением http://www.mathnet.ru/rus/agreement

Параметры загрузки:

IP : 18.208 .226 .222

26 апреля 2023 г., $17: 40: 53$

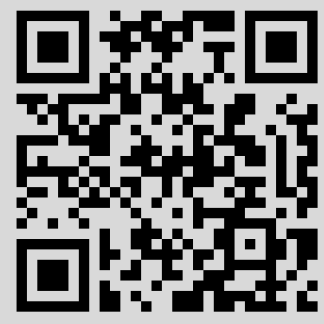




\section{О существовании целых решений одного полулинейного эллиптического уравнения на некомпактных римановых многообразиях}

\section{Е. А. Мазепа}

Проблемы существования целых положительных решений полулинейных эллиптических уравнений для неограниченных областей в $\mathbb{R}^{n}$ рассматривались в работах ряда авторов [1]-[5]. Вопросы существования решений подобных уравнений в ограниченных областях $\mathbb{R}^{n}$ достаточно полно изучены в монографиях Е. М. Ландиса [6], О. А. Ладыженской, Н. Н. Уральцевой [7], Д. Гилбарга, М. Трудингера [8].

Однако вопросы существования целых решений полулинейных эллиптических уравнений на некомпактных и особенно полных римановых многообразиях оставались открытыми.

Пусть $M$ - произвольное гладкое связное некомпактное риманово многообразие без края, $B \subset M$ - произвольное связное компактное подмножество, граница $\partial B$ - гладкое подмногообразие в $M$ и $M \backslash B$ связно. Обозначим $\left\{B_{k}\right\}_{k=1}^{\infty}-$ исчерпание многообразия $M$, т.е. последовательность предкомпактных открытых подмножеств риманова многообразия $M$ таких, что $\bar{B}_{k} \subset B_{k+1}, M=\cup_{k=1}^{\infty} B_{k}$ и пусть $B \subset B_{k}$ для всех $k$.

Говорят, что на многообразии $M$ выполнено лиувиллево свойство для уравнения

$$
\Delta u-u=0,
$$

если любое ограниченное решение этого уравнения тождественно равно нулю.

Аналогично, будем говорить, что на многообразии $M$ выполнено лиувиллево свойство для уравнения

$$
\Delta u=u \varphi(|u|),
$$

где $\varphi(\xi)>0$ - монотонно неубывающая, непрерывно дифференцируемая функция при $0 \leqslant \xi<\infty$, если любое ограниченное решение этого уравнения тождественно равно нулю.

Известно (см. [9], [10]), что стохастическая неполнота некомпактного риманового многообразия (риманово многообразие стохастически полное, если стохастический процесс на нем имеет бесконечное время жизни) эквивалентна существованию на нем нетривиального ограниченного решения уравнения (1). Причем из существования нетривиального ограниченного решения для уравнения (1) следует существование аналогичного решения для уравнения $\Delta u-\mu u=0$, где $\mu>0-$ произвольная константа.

В данной работе изучаются вопросы взаимосвязи выполнения лиувиллевых свойств для уравнений (1) и (2) на некомпактных римановых многообразиях, и как следствие, вопросы существования на рассматриваемых многообразиях нетривиальных целых ограниченных решений для полулинейного эллиптического уравнения (2).

ЛЕмма 1. Если на М существует нетривиалъное ограниченное решение уравнения (2), то на $М$ существует и положительное ограниченное решение этого уравнения.

Доказательство. Пусть $u_{0} \not \equiv 0$ - ограниченное решение уравнения (2) на $M$. Покажем, что на $M$ существует положительное ограниченное решение этого уравнения.

Обозначим $a^{+}=\max \{0, a\}$. Рассмотрим последовательность функций $\left\{u_{k}\right\}_{k=1}^{\infty}$, являющихся решением задачи

$$
\left\{\begin{array}{l}
\Delta u_{k}=u_{k} \varphi\left(\left|u_{k}\right|\right)=0 \quad \text { в } B_{k}, \\
\left.u_{k}\right|_{\partial B_{k}}=\left.u_{0}^{+}\right|_{\partial B_{k}} .
\end{array}\right.
$$

Можем считать, что $u_{0}^{+} \not \equiv 0$. Тогда, учитывая принцип максимума, для всех $k$ имеем $0 \leqslant u_{k} \leqslant \sup _{M} u_{0}^{+}$. 
Так как

$$
\Delta u_{k}=u_{k} \varphi\left(\left|u_{k}\right|\right), \quad \Delta u_{0}=u_{0} \varphi\left(\left|u_{0}\right|\right),\left.\quad u_{k}\right|_{\partial B_{k}} \geqslant\left. u_{0}\right|_{\partial B_{k}},
$$

то, применяя принцип сравнения для решений полулинейных эллиптических уравнений (см., например, [11]), получаем $u_{k} \geqslant u_{0}$ в $B_{k}$ и, следовательно, $u_{k} \geqslant u_{0}^{+}$в $B_{k}$ для всех $k$.

Кроме того, из равномерной ограниченности последовательности $\left\{u_{k}\right\}_{k=1}^{\infty}$ на любом компактном подмножестве многообразия $M$ следует существование предельной функции $u=\lim _{k \rightarrow \infty} u_{k}$, которая является ограниченным положительным решением уравнения (2) на $M$ (см., [11]). Лемма доказана.

ЗАмечАниЕ 1. Аналогичное утверждение для решений уравнения (1) было получено в [12].

Теорема 1. На М выполнена лиувиллева теорема для уравнения (2) тогда и только тогда, когда на $M$ выполнена лиувиллева теорема для уравнения (1).

ДокАзАТЕльство. Так как $\varphi(\xi)$ - монотонно неубывающая и положительная при $\xi \geqslant 0$ функция, существует константа $A>0$ такая, что $A \varphi(0) \geqslant 1$ и, следовательно, $A \varphi(\xi) \geqslant 1$ для всех $\xi \geqslant 0$. Без ограничения общности можем считать, что $A \geqslant 1$.

Докажем достаточность. Пусть существует функция $u_{0} \not \equiv 0$ - ограниченное решение уравнения (2) на $M$. Можем считать, что $u_{0}$ - положительное ограниченное решение уравнения (2). Покажем, что на $M$ существует положительное ограниченное решение уравнения

$$
\Delta u=A u \varphi(|u|)
$$

Рассмотрим решения следующих краевых задач:

$$
\left\{\begin{array}{l}
\Delta u_{k}=A u_{k} \varphi\left(\left|u_{k}\right|\right) \quad \text { в } B_{k}, \\
\left.u_{k}\right|_{\partial B_{k}}=\left.u_{0}\right|_{\partial B_{k}} .
\end{array}\right.
$$

Так как $0 \leqslant u_{k} \leqslant \sup _{M} u_{0}$, существует функция $u=\lim _{k \rightarrow \infty} u_{k}$, причем $0 \leqslant u \leqslant$ $\sup _{M} u_{0}$. Кроме того, $\Delta u_{0}=u_{0} \varphi\left(\left|u_{0}\right|\right) \leqslant A u_{0} \varphi\left(\left|u_{0}\right|\right)$; тогда с учетом принципа сравнения в $B_{k}$ выполнено $0 \leqslant u_{k} \leqslant u_{0}$ и, следовательно, $0 \leqslant u \leqslant u_{0}$. Покажем, что $u \not \equiv 0$.

Рассмотрим функции $w_{k}, \bar{u}_{k}, \bar{v}_{k}$, которые являются решениями соответствующих краевых задач в области $B_{k}$ :

$$
\left\{\begin{array} { l } 
{ \Delta w _ { k } = 0 , } \\
{ w _ { k } | _ { \partial B _ { k } } = u _ { 0 } | _ { \partial B _ { k } } , }
\end{array} \quad \left\{\begin{array} { l } 
{ \Delta \overline { v } _ { k } = - u _ { 0 } \varphi ( | u _ { 0 } | ) , } \\
{ \overline { v } _ { k } | _ { \partial B _ { k } } = 0 , }
\end{array} \quad \left\{\begin{array}{l}
\Delta \bar{u}_{k}=-A u_{k} \varphi\left(\left|u_{k}\right|\right), \\
\left.\bar{u}_{k}\right|_{\partial B_{k}}=0 .
\end{array}\right.\right.\right.
$$

Ясно, что $\Delta w_{k}=\Delta\left(u_{k}+\bar{u}_{k}\right)=0$ и $\left.w_{k}\right|_{\partial B_{k}}=\left.\left(u_{k}+\bar{u}_{k}\right)\right|_{\partial B_{k}}=\left.u_{0}\right|_{\partial B_{k}}, \Delta w_{k}=\Delta\left(u_{0}+\bar{v}_{k}\right)=0$ и $\left.w_{k}\right|_{\partial B_{k}}=\left.\left(u_{0}+\bar{v}_{k}\right)\right|_{\partial B_{k}}=\left.u_{0}\right|_{\partial B_{k}}$. Тогда по теореме единственности для гармонических функций в каждой области $B_{k}$ выполнено $w_{k}-\bar{u}_{k}=u_{k}, w_{k}-\bar{v}_{k}=u_{0}$; кроме того, $u_{0} \leqslant w_{k} \leqslant \sup _{M} u_{0}, \bar{u}_{k} \geqslant 0, \bar{v}_{k} \geqslant 0$. Покажем, что $\bar{u}_{k} \leqslant A \bar{v}_{k}$.

Действительно,

$$
\Delta\left(A \bar{v}_{k}\right)=-A u_{0} \varphi\left(\left|u_{0}\right|\right) \leqslant-A u_{k} \varphi\left(\left|u_{k}\right|\right)=\Delta \bar{u}_{k}
$$

тогда из принципа сравнения получаем $A \bar{v}_{k} \geqslant \bar{u}_{k}$.

Далее, выберем точку $x_{0}$, в которой $u_{0}\left(x_{0}\right)>\sup _{M} u_{0}-\varepsilon$. Тогда

$$
\begin{gathered}
w_{k}\left(x_{0}\right)>\sup _{M} u_{0}-\varepsilon, \\
\bar{v}_{k}\left(x_{0}\right)=w_{k}\left(x_{0}\right)-u_{0}\left(x_{0}\right) \leqslant \sup _{M} u_{0}-u_{0}\left(x_{0}\right)<\varepsilon, \\
\bar{u}_{k}\left(x_{0}\right)<A \varepsilon, \quad u_{k}\left(x_{0}\right)=w_{k}\left(x_{0}\right)-\bar{u}_{k}\left(x_{0}\right)>\sup _{M} u_{0}-(A+1) \varepsilon .
\end{gathered}
$$


Тогда при $k \rightarrow \infty$ и достаточно малом $\varepsilon>0$ получаем $u\left(x_{0}\right) \geqslant \sup _{M} u_{0}-(A+1) \varepsilon>0$. Таким образом, функция $u$ является положительным ограниченным решением уравнения (3).

Покажем теперь, что на $M$ существует положительное ограниченное решение уравнения (1).

Рассмотрим последовательность функций $\left\{u_{k}\right\}_{k=1}^{\infty}$, являющихся решением задачи

$$
\left\{\begin{array}{l}
\Delta u_{k}-u_{k}=0 \\
\left.u_{k}\right|_{\partial B_{k}}=\left.u\right|_{\partial B_{k}} .
\end{array} \text { в } B_{k},\right.
$$

Тогда, учитывая принцип максимума, для всех $k$ имеем $0 \leqslant u_{k} \leqslant \sup _{M} u$.

Так как

$$
\Delta u_{k}=u_{k} \leqslant A u_{k} \varphi\left(\left|u_{k}\right|\right), \quad \Delta u=A u \varphi(|u|),\left.\quad u_{k}\right|_{\partial B_{k}}=\left.u\right|_{\partial B_{k}},
$$

применяя принцип сравнения для решений полулинейных эллиптических уравнений (см., например, [11]), получаем $u_{k} \geqslant u>0$ в $B_{k}$.

Из равномерной ограниченности последовательности $\left\{u_{k}\right\}_{k=1}^{\infty}$ на любом компактном подмножестве многообразия $M$ следует существование предельной функции для этой последовательности, которая является ограниченным положительным решением уравнения (1) на $M$, что противоречит условию.

Необходимость. Пусть теперь на $M$ существует нетривиальное ограниченное решение уравнения (1). Тогда на $M$ существуют положительное ограниченное решение этого уравнения $v_{0}$ (см., например, [12]).

Положим $C=\sup _{M} v_{0}>0$. Покажем сначала, что на $M$ существует положительное ограниченное решение уравнения (3).

Как и выше рассмотрим решения следующих краевых задач:

$$
\left\{\begin{array}{l}
\Delta u_{k}=A u_{k} \varphi\left(\left|u_{k}\right|\right) \quad \text { в } B_{k}, \\
\left.u_{k}\right|_{\partial B_{k}}=\left.v_{0}\right|_{\partial B_{k}} .
\end{array}\right.
$$

Так как $0 \leqslant u_{k} \leqslant \sup _{M} v_{0}$, существует функция $u=\lim _{k \rightarrow \infty} u_{k}$, причем $0 \leqslant u \leqslant C$. Кроме того, $\Delta v_{0}=v_{0} \leqslant A v_{0} \varphi\left(\left|v_{0}\right|\right)$; тогда с учетом принципа сравнения в $B_{k}$ выполнено $0 \leqslant u_{k} \leqslant v_{0}$ для любого $k$ и, следовательно, $0 \leqslant u \leqslant v_{0}$. Покажем, что $u \not \equiv 0$.

Рассмотрим функции $w_{k}, \bar{u}_{k}, \bar{v}_{k}$, которые являются решениями соответствующих краевых задач в области $B_{k}$ :

$$
\left\{\begin{array} { l } 
{ \Delta w _ { k } = 0 , } \\
{ w _ { k } | _ { \partial B _ { k } } = v _ { 0 } | _ { \partial B _ { k } } , }
\end{array} \quad \left\{\begin{array} { l } 
{ \Delta \overline { v } _ { k } = - v _ { 0 } , } \\
{ \overline { v } _ { k } | _ { \partial B _ { k } } = 0 , }
\end{array} \quad \left\{\begin{array}{l}
\Delta \bar{u}_{k}=-A u_{k} \varphi\left(\left|u_{k}\right|\right), \\
\left.\bar{u}_{k}\right|_{\partial B_{k}}=0 .
\end{array}\right.\right.\right.
$$

Тогда, как и при доказательстве достаточного условия, по теореме единственности имеем $u_{k}=w_{k}-\bar{u}_{k}, v_{0}=w_{k}-\bar{v}_{k}$, причем $\Delta v_{0}=v_{0}>0$ и, следовательно, $v_{0} \leqslant w_{k} \leqslant C, \bar{u}_{k} \geqslant 0$, $\bar{v}_{k} \geqslant 0$.

\section{Найдем}

$$
\Delta\left(\bar{u}_{k}\right)=-A u_{k} \varphi\left(\left|u_{k}\right|\right) \geqslant-A v_{0} \varphi\left(\left|v_{0}\right|\right) \geqslant-A v_{0} \varphi(C)=\Delta\left(A \varphi(C) \bar{v}_{k}\right) .
$$

Кроме того, $\left.\bar{u}_{k}\right|_{\partial B_{k}}=\left.\bar{v}_{k}\right|_{\partial B_{k}}=0$; тогда из принципа сравнения получаем $\bar{u}_{k} \leqslant A \varphi(C) \bar{v}_{k}$.

Далее, выберем точку $x_{0}$, в которой $v_{0}\left(x_{0}\right)>\sup _{M} v_{0}-\varepsilon=C-\varepsilon$. Тогда

$$
\begin{gathered}
w_{k}\left(x_{0}\right) \geqslant v_{0}\left(x_{0}\right)>\sup _{M} v_{0}-\varepsilon, \\
\bar{v}_{k}\left(x_{0}\right)=w_{k}\left(x_{0}\right)-v_{0}\left(x_{0}\right) \leqslant C-v_{0}\left(x_{0}\right)<\varepsilon, \\
\bar{u}_{k}\left(x_{0}\right) \leqslant A \varphi(C) \bar{v}_{k}\left(x_{0}\right)<A \varphi(C) \varepsilon, \\
u_{k}\left(x_{0}\right)=w_{k}\left(x_{0}\right)-\bar{u}_{k}\left(x_{0}\right)>C-\varepsilon-A \varphi(C) \varepsilon=C-\varepsilon(1+A \varphi(C)) .
\end{gathered}
$$


Тогда при $k \rightarrow \infty$ получаем $u\left(x_{0}\right) \geqslant C-\varepsilon(1+A \varphi(C))$, что больше нуля при достаточно малом $\varepsilon>0$. Функция $u$ является положительным ограниченным решением уравнения (3).

Так как $A \geqslant 1$, для любого $\xi \geqslant 0$ выполнено $\varphi(\xi) \leqslant A \varphi(\xi)$. Известно (см., [11]), что из существования положительного ограниченного решения для уравнения (3) следует существование аналогичного решения для уравнения (2), что противоречит условию. Теорема доказана.

СлЕДСтвиЕ 1. На многообразии $M$ существуют нетривиальные ограниченные решения уравнения (2) тогда и только тогда, когда $M$ не является стохастически полным.

СлЕДСТвИЕ 2. Если многообразия $M_{1}$ и $M_{2}$ изометричны вне некоторого компакта, то лиувиллево свойство для уравнения (2) на $M_{1}$ и $M_{2}$ выполнено или нет одновременно.

ДокАЗАТЕЛЬСтво непосредственно следует из теоремы. Достаточно заметить (см. [12]), что если многообразия $M_{1}$ и $M_{2}$ изометричны вне некоторого компакта, то они стохастически полны или нет одновременно.

СледСтвиЕ 3. Если многообразия $M_{1}$ и $M_{2}$ изометричны вне некоторого компакта, то иелые положительные ограниченные решения уравнения (2) на $M_{1}$ и $M_{2}$ существуют или нет одновременно.

ДоКАЗАТЕЛЬСтво получаем путем непосредственного применения следствия 2 и следующего замечания.

ЗАмечАние 2 . Пусть $G_{1}, G_{2}$ - некоторые предкомпактные области многообразия $M$ такие, что $B \subset G_{1}$ и $\bar{G}_{1} \subset G_{2}$. Обозначим $M_{i}(v)=\sup _{\partial G_{i}} v, m_{i}(v)=\inf _{\partial G_{i}} v$. На $M$ существует целое положительное ограниченное решение $u(x)$ уравнения (2) тогда и только тогда, когда на $M \backslash B$ существует целое положительное ограниченное решение $v(x)$ этого уравнения, удовлетворяющее условиям $M_{1}(v)<M_{2}(v), m_{i}(v)>0, i=1,2$.

Данное утверждение является частным случаем аналогичного утверждения, справедливого для произвольных ненулевых (не обязательно положительных) ограниченных решений уравнения (2) (см. [11]).

\section{СПИСОК ЦИТИРОВАННОЙ ЛИТЕРАТУРЫ}

[1] Е. М. Ландис, Нелинейные граничные задачи, 1991, № 3, 49-53. [2] J.-L. Vazquez, L. Veron, C. R. Acad. Sci. Paris Sér. I, 312:11 (1991), 811-815. [3] M. Garcia-Huidobro, R. Manasevich, P. Ubilla, Electronic J. Differential Equations, 1:10 (1995), 1-22. [4] Y. Naito, H. Usami, Math. Z., 225:1 (1997), 167-175. [5] L. Ambrosio, X. Cabre, J. Amer. Math. Soc., 13:4 (2000), 725-739. [6] Е. М. Ландис, Уравнения второго порядка эллиптического u параболического типов, Наука, М., 1971. [7] О. А. Ладыженская, Н. Н. Уральцева, Линейные и квазилинейные уравнения эллиптического типа, 2-е изд., Наука, М., 1973. [8] Д. Гилбарг, М. Трудингер, Эллиптические дифференииальные уравнения с частными производными второго порядка, Наука, М., 1989. [9] A. Grigor'yan, Bull. Amer. Math. Soc., 36:2 (1999), 135-249. [10] E. B. Davies, Bull. London Math. Soc., 17:5 (1985), 417-436. [11] Е. А. Мазепа, Изв. вузов. Матем., 3 (2005), 59-66. [12] А. А. Григорьян, Н. С. Надирашвили, Изв. вузов. Матем., 1987, № 5, 25-33.

\section{Е. А. Мазепа}

Поступило

Волгоградский государственный университет

12.12 .2005

E-mail: imazepa@rambler.ru 